\title{
Instalación e Implementación de DSpace como Repositorio Institucional de Documentos Científicos en la Universidad Nacional de Jaén (UNJ).
}

\author{
Installation and deployment of DSpace as Institutional Repository \\ of Scientific Documents at the National University of Jaén. \\ ${ }^{1}$ Mack Johan Tarrillo Herrera y ${ }^{2} J o s e ́$ Willian Tarrillo Gaona
}

\section{RESUMEN}

Los repositorios digitales, posibilitan una gestión automatizada de la producción administrativa, académica o científica de una institución, facilitando de forma ordenada la difusión y accesibilidad de sus contenidos a través de internet. El objetivo de este artículo es proyectar una visión general para instalar e implementar un Repositorio de documentos científicos y de investigación en la Universidad Nacional de Jaén (UNJ) mediante la plataforma DSpace. La metodología tomada en cuenta para desarrollar la propuesta incluyó la realización de un diagnóstico en la UNJ a través de cuestionarios digitales para identificar y conocer cómo se preservan los documentos científicos, tesis, entre otros; además se instaló el Repositorio Institucional para la conservación y difusión de trabajos de investigación a partir de la herramienta Dspace. El Repositorio se adecuó a los lineamientos de RENATI (Registro Nacional de Trabajos de Investigación) a las normas de la Universidad Nacional de Jaén; de igual forma, detallar la instalación, mantenimiento y desarrollo del Repositorio Institucional en esta casa universitaria. Este trabajo pretende ser un aporte para las instituciones que buscan difundir su producción académica y científica a nivel mundial, incentivando a gestionar, preservar y difundir trabajos de investigación que se utilizarán como referentes para futuras investigaciones.

Palabras clave: Software libre, repositorio digital, plataforma, DSpace.

\begin{abstract}
The digital repositories, allow an automated management, of the administration of academic scientific and production of an institution, facilitating in an orderly way the diffusion and accessibility of its contents through internet. The aim of this article is to project a general vision for implementing a Repository of scientific research documents at Jaen National University (JNU) through the DSpace platform. The methodology used to develop this proposal included a diagnosis at JNU through digital questionnaires to identify and to know how scientific documents, theses, among others; are preserved. In addition, the Institutional Repository for the preservation and dissemination of research work from the DSpace tool, was designed: the repository was adequated to the guidelines of RENATI (National Registry of Research Works) and the rules of the of JNU. It is also detailed the installation, maintenance and development of the Institutional Repository in this university. This research aims to be a contribution for institutions that seek to disseminate their academic and scientific production worldwide, encouraging them to manage, preserve and disseminate research that will be used as references in the future.
\end{abstract}

Keywords: Free software, digital repository, platform, Dspace.

\footnotetext{
${ }^{1}$ Universidad Nacional de Jaén. Jaén, Cajamarca, Perú

${ }^{2}$ I.E. Cristo Rey - Fila Alta. Jaén, Cajamarca, Perú
} 


\section{INTRODUCCIÓN}

En la actualidad, la educación integral del ser humano se encuentra volcada en una búsqueda constante de información y conocimiento, interactuando de manera cotidiana con el internet y sus motores de búsqueda de información; un gran ejemplo es Google que procesa alrededor de 3 billones de búsquedas por día, lo que significan 90 billones de búsquedas al mes(Silva, 2015). Es por ello que en la actualidad se está dejando de lado la búsqueda y consulta de información tradicional que consiste en la relación lector biblioteca. Conscientes de esta realidad originada a consecuencia de los avances tecnológicos e informáticos, han surgido un sin número de medios y herramientas para reunir, preservar y difundir producción científica y académica de forma virtual, resultando como respuesta para muchas instituciones, los Repositorios Digitales.

Los Repositorios Digitales son medios que nos permiten gestionar, almacenar, preservar, difundir y facilitar el acceso a los objetos digitales que este alberga (Polanco, 2016).

Algunas de las herramientas en software libre más utilizadas para implementar un repositorio son las siguientes: DSpace, E-print, LUCENE, Protocolo OAI-PMH, etc. Estos recursos facilitan a las instituciones públicas como privadas, la instalación, implementación y mantenimiento de repositorios digitales, para organizar y administrar la información de forma virtual, información que está conformada básicamente por documentos como informes, proyectos, artículos, etc., teniendo en las instituciones académicas las más recurrentes en utilizar estas herramientas.

La herramienta para gestión de objetos digitales DSpace se ha posicionado en este marco como una de las aplicaciones más extendida y mejor valorada tanto por las instituciones públicas como por las organizaciones privadas, avalado por las 582 instalaciones existentes en España, las 302 instalaciones presentes en Europa y las
835 en total a nivel internacional (Ibai Sistemas S.A., 2012).

DSpace es un software de código abierto diseñado por el Massachusetts Institute of Technology (MIT) y los laboratorios de HP para gestionar repositorios de ficheros (textuales, audio, vídeo, etc.), facilitando su depósito, organizándolos en comunidades, asignándoles metadatos y permitiendo su difusión a recolectores o agregadores. Estas características han hecho que, junto con EPrints, sea uno de los programas preferidos por las instituciones académicas para gestionar el repositorio dónde los investigadores depositan sus publicaciones y materiales de búsqueda con objeto de darles una mayor visibilidad.(Rodríguez \& Sulé, 2008).

En el Perú, existen noventa instituciones que cuentan con repositorios digitales implementados y asociados al Repositorio Nacional Digital de Acceso Abierto - ALICIA, tal como exige SUNEDU en sus Condiciones Básicas de Calidad para el licenciamiento de las Universidades Peruanas.

La Universidad Nacional de Jaén, no contaba con un repositorio que maneje estos recursos, es por ello que se presenta esta propuesta que consiste en instalar un Repositorio Institucional para reunir, preservary difundir producción científica y académica de forma virtual, utilizando la herramienta de software libre DSpace, teniendo como objetivos principales los siguientes:

a) Realizar un estudio de la herramienta DSpace para la instalación de un repositorio digital.

b) Presentar una propuesta de instalación, desarrollo y mantenimiento de un Repositorio Institucional de almacenamiento de documentos científicos y académicos producidos en la UNJ.

\section{MATERIALES Y MÉTODOS Tipo de estudio}

Para el desarrollo del presente trabajo se realizó una investigación Descriptiva y aplicada; descriptiva porque se analizará datos recopilados a través de instrumentos estadísticos y de tipo 
aplicada por ser una implementación a realizarse en las instalaciones de la UNJ para la gestión y preservación de sus documentos digitales de carácter científico y académico. Este trabajo se desarrolló en tres etapas que serán detalladas a continuación.

\section{Primera etapa: EI Problema}

\subsection{Problemática de la UNJ}

Según el organigrama oficial, la Universidad Nacional de Jaén, cuenta con nueve unidades de investigación entre las cuales se encuentran: Dirección de Gestión de Información y Transferencia tecnológica, Dirección General de Investigación e Innovación, Instituto Binacional de Investigación para el Desarrollo Sustentable de la cuenca del Río Marañón de Perú y Ecuador (IBIDESPE), entre otras, que generan diversos documentos científicos y administrativos necesarios para la gestión de la institución, producidos en diferentes procesadores de texto que después de ser impresos pasan por las distintas oficinas según el flujo y el trámite regular establecido en la UNJ, para luego ser distribuidos y/o guardados en archivos físicos de cada unidad. Por ello, se solicitó a la Vicepresidencia de Investigación los trabajos científicos y/o Proyectos de investigación que tuvieran disponibles del año 2016 obteniendo el resultado de 15 documentos de proyectos de tesis. Posteriormente se aplicó un cuestionario, para relevar las opiniones de los responsables de la gestión administrativa de la Vicepresidencia de Investigación y generar un desarrollo acorde con las expectativas de la institución. Este cuestionario se realizó vía internet mediante la herramienta Google Forms (octubre del 2016) a tres directores o jefes activos de las distintas unidades de la Vicepresidencia de Investigación y sus respectivas secretarias (tres), para que dieran información respecto a la tipología documental (proyectos de tesis, trabajos de investigación, artículos), los modos de almacenamiento y respaldo realizados en las distintas dependencias.

En concreto, la información recabada en el cuestionario que se analizará al detalle en la sección de "resultados" de este artículo y la experiencia como administrativo y Docente de la UNJ, me permite afirmar que las unidades de Vicepresidencia de Investigación necesitan un centro de archivo y almacenamiento digital, que admita preservar, difundir y facilitar la búsqueda de documentos científicos y administrativos; puesto que esta información se encuentra almacenada físicamente en estantes, archivadores y en algunos casos en USB/CD, lo que ocasiona numerosos problemas como el tiempo excesivo de localización, destrucción por alguna actividad natural o humana como: incendios, inundaciones, pérdida de documentos, entre otras; provocando un grave problema institucional en la preservación de documentos.

\section{Diseño de la Propuesta}

La propuesta está definida sobre una solución concreta para archivar, preservar y difundir trabajos de investigación finales producidos por la Vicepresidencia de Investigación de la UNJ, desarrollándose bajo el conocimiento de Software Libre y Open Access, sin dejar de cumplir con los lineamientos establecidos por RENATI (Registro Nacional de Trabajos de Investigación) y el reglamento de la UNJ. Si bien este trabajo fue realizado para la UNJ que cuenta con Comisión Organizadora, podría adaptarse a cualquier otra universidad o institución peruana. Para el desarrollo del Repositorio Institucional UNJ, se determinó trabajar con DSpace como herramienta de gestión de repositorios digitales, por presentar facilidad en el desarrollo y gestión de colecciones de recursos digitales, locales o distribuidos, sin restricción de formato, además de sus productos y facilidades creados para dar valor al contenido de la colección, adecuados a las necesidades y a los requisitos de sus usuarios (Chazarra, Requena, \& Valverde, 2010). 
La implementación del servidor DSpace se hizo en un equipo perteneciente a la UNJ y se encuentra ubicado en el ambiente de servidores de esta alma máter; posteriormente el Repositorio Institucional fue evaluado por las autoridades de la UNJ y luego por el Repositorio Nacional Digital de Acceso Abierto - ALICIA para una posterior asociación.

\section{Segunda etapa: Análisis, Instalación y Configuración}

Esta etapa se comprendió un análisis exhaustivo de la problemática en la UNJ, instalación e implementación de un Repositorio Institucional, con el objetivo de dar una solución al problema planteado.

\subsection{Planificación}

En estos últimos años ALICIA - CONCYTEC ha tomado una participación protagónica con el tema de la implementación de repositorios digitales en las universidades públicas y privadas, estableciendo directrices para la creación de estos repositorios, estableciendo los siguientes criterios: (ALICIA - CONCYTEC, 2016):

a) El repositorio institucional debe contar con un subdominio propio.

b) Las revistas que se integren al Repositorio Nacional deben estar indizadas en el Sistema Latindex y contar con una plataforma de gestión que contenga el protocolo OAI-PMH para su integración e interoprabilidad con el Repositorio Nacional.

c) Información de derechos de autor legible para las máquinas (ejem. Creative Commons).

d) Publicar en el repositorio institucional las políticas de publicación.

e) Registrar el repositorio institucional en el directorio OpenDoar (www.opendoar.org).

f) Implementar módulo de estadísticas de acceso a los contenidos de manera visible.

Estos criterios son básicos para las instituciones que desean ser asociadas a ALICIA CONCYTEC. Cabe señalar que este trabajo se limita a señalar los pasos para una instalación de DSpace en la versión 5.6, configuración básica, mostrar su estructura y archivos para un trabajo mínimo y las opciones de catalogación de los documentos.

\subsection{Instalación}

El ambiente de desarrollo estará de acorde con las guías de instalación de software de repositorios propuesto por ALICIA (ALICIA CONCYTEC, 2016), donde se recomienda los requisitos mínimos en hardware y software. A continuación, se detallará la configuración mínima para la implementación de un repositorio digital con DSpace 5.6 que cumpla con las exigencias de ALICIA - CONCYTEC.

\section{Hadware:}

a) Memoria 4GB, recomendable 8GB.

b) Almacenamiento: $250 \mathrm{~GB}$.

\section{Software}

a) Sistema Operativo Ubuntu 12.04.

b) OpenJDK 7.

c) Apache Maven 3.x.

d) Apache Ant 1.8 o superior.

e) PostgreSQL (9.x) o superior.

f)Apache Tomcat 7 o superior.

De acuerdo con las experiencias recogidas durante el período de prueba y evaluación que tuvo nuestro repositorio institucional y las recomendaciones hechas por ALICIA, para un mejor desarrollo y mantenimiento del repositorio digital se sugiere la siguiente estructura:

1. /build

2. /dspace

3. /dspace/config
a. /dspace/config/dspace.cfg

4. /dspace/webapps
a. /dspace/webapps/jspui
b. /dspace/webapps/oai
c. /dspace/webapps/xmlui 
La estructura tiene un diseño que se enfoca a mostrar una plataforma amigable para los usuarios que accederán a nuestro repositorio, igualmente posee dos entornos a elegir y establecer como index o plataforma principal para mostrarse al público en general, algunas configuraciones vienen predeterminadas en la instalación de DSpace pero otras configuraciones como por ejemplo el idioma, los metadatos, entre otros; se realizan en archivos con extensión .xml contenidas en las carpetas de la estructura antes descrita. A continuación, se detallará de manera breve la funcionalidad de la estructura Dspace:

- /build: Esta carpeta se creará en el sistema operativo, donde se se guardará el paquete DSpace 5.6 para luego desempaquetaremos con mvn y originar el instalador.

- /dspace: Esta carpeta creada en el sistema operativo alojará los archivos generados a efectos del desempaquetamiento con mvn y luego se instalará DSpace 5.6

- /dspace/config: Directorio que contiene alojados los archivos a configurar DSpace como repositorio.

- /dspace/config/dspace.cfg: Archivo principal para configurar el idioma, metadatos, entre otros.

- /dspace/webapps: Este directorio aloja los diversos index y presentaciones a mostrar a los usuarios.

- /dspace/webapps/jspui: En este directorio se ubicarán todos los archivos correspondientes a la interfaz clásica de DSpace, esta interfaz usa JSP y Java Servlet API.

- /dspace/webapps/oai: Básicamente este directorio almacena los archivos que ayudan a facilitar la adecuación del Repositorio a los aspectos derivados de la recolección y minería de datos.

- /dspace/webapps/xmlui: Directorio que contiene los archivos correspondientes a la interfaz XMLUI la cual funciona con Apache Cocoon y usa XML y XSLT, cabe señalar que el Repositorio Institucional de la Universidad Nacional de Jaén usará esta interfaz para sus usuarios.
Es muy importante señalar que en el servidor físico donde se instalará DSpace, debe contar con la funcionalidad correcta del servidor web (Tomcat 7) y el sistema se debe encontrar accesible al administrador. De la misma forma la base de datos (PostgreSQL) tiene que contar con una funcionalidad estable y correcta, además de realizar la creación de su usuario o usuarios que tendrán acceso a la base de datos del Repositorio Institucional.

Las líneas de comandos para una instalación rápida en el terminal del Sistema Operativo Ubuntu 12.04, se realizará de acuerdo a las recomendaciones hechas por DURASPACE. A continuación, se dejará las líneas de comando para una instalación rápida de DSpace 5.6 (DURASPACE, 2016).

1. useradd -m dspace.

2. gzip xzf dspace-5.x-src-release.tar.gz

3. createuser - -username $=$ postgres - -no superuser --pwprompt dspace.

4.createdb --username=postgres -owner $=$ dspace - -encoding $=$ UNICODE dspace.

5. cd [dspace-source]

6. vi build.properties

7. mkdir [dspace]

8. chown dspace [dspace]

9. su-dspace

10. cd [dspace-source]

11. mvn package

12. cd [dspace-source]/dspace/target/dspaceinstaller

13. ant fresh_install

14. cp -r [dspace]/webapps/* [tomcat]/webapps

15. /etc/init.d/tomcat start

16. [dspace]/bin/dspace create-administrator

\subsection{Configuración}

Para una configuración de DSpace que cumpla con las directrices de CONCYTEC y RENATI, utilizaremos los parámetros configurables establecidas por ALICIA. Los parámetros y metadatos a configurar son las siguientes (ALICIA, 2016): 
a) Direccionar el sub dominio de:

http://repositorio.dominio de institucion:8080/is

pui

- $\mathrm{O}$ en el caso de:

http://repositorio.dominio de institucion:8080/x mlui.

-Alo siguiente:

http://repositorio.dominio_de_institucion en este caso para el repositorio Institucional de la UNJ se determinó usar http://repositorio.unj.edu.pe.

b) Modificar el archivo dspace.cfg ubicado en /[dspace_compilado]/config/, de la siguiente manera:

-\#dspace.hostname $=$ localhost

-\# Modificar el subdominio de su institución

- dspace.hostname =

repositorio.concytec.gob.pe

-\#dspace.baseUrl = http://localhost:8080

- \# Modificar el subdominio de su institución

- dspace. baseUrl =

http://repositorio.concytec.gob.pe

- \#dspace.url = http://localhost:8080/xmlui

- \# Modificar el subdominio de su institución

- dspace.url =

http://repositorio.concytec.gob.pe

- \#dspace.name = DSpace at My University

- \# Modificar el nombre del repositorio

- dspace.name $=$ Repositorio

institucional de la Universidad

- \# default.language $=$ en_US

- \# Modificar el idioma de los metadatos

- default.language $=$ es_ES

- \# handle.canonical.prefix $=$

http://hdl.handle.net/

- \#Modificar el identificador, no olvidar cerrar el handle/

- handle.canonical.prefix $=$

http://repositorio.concytec.gob.pe/handle/

- \# handle.prefix $=123456789$

- \# Modificar el código con las iniciales de su institución

handle.prefix $=$ CONCYTEC

- \# default.locale $=$ en
-\# Modificar el idioma del sistema

- default.locale $=$ es

-\#Agregar la localidad del idioma

webui.supported.locales $=$ es

c) Modificar el archivo oai.cfg ubicado en /[dspace_compilado]/config/modules/, de la siguiente forma:

identifier.prefix $=$ repositorio.concytec.gob.pe bitstream.baseUrl = http://repositorio.concytec.gob.pe d) Modificar el archivo input-forms.xml ubicado en /[dspace_compilado]/config/, en este caso la modificación se tendrá que hacer con programación en xml y se hará de acuerdo a las necesidades de Universidades, Instituciones y Organizaciones que implementarán su repositorio digital.

e) Por último, modificaremos la hoja de estilo de DSpace, en el archivo style.css ubicado en /[dspace_compilado]/webapps/xmlui/themes/M irage/lib/css/, en este caso las modificaciones quedarán al criterio del administrador del repositorio digital.

\section{RESULTADOS}

Como se mencionó con anterioridad se aplicó una encuesta digital con la herramienta gratuita Google Forms (octubre del 2016) a dos directores y un jefe de las distintas unidades de la Vicepresidencia de Investigación (Incubadora de empresas, IBIDESPE y la Oficina de Gestión de investigación) y sus respectivas secretarias (tres), arrojó los siguientes resultados:

El $66.6 \%$ de las personas que completaron el cuestionario realizan respaldos de los documentos digitales a través de USB, CD, DVD y/o en la misma computadora, el $33.4 \%$ no realizan respaldo alguno. (Figura. 1)

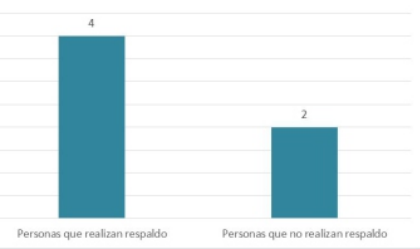

Figura 1. Personas que realizan respaldo de documentos digitales. 
b) El $16.67 \%$ de las personas que completaron el cuestionario archivan los documentos físicos producidos en los últimos 5 años. (Figura 2)

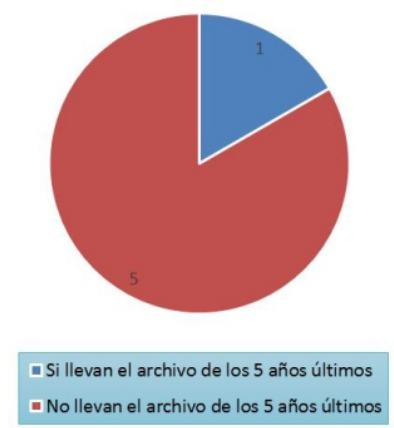

Figura 2. Archivamiento de documentos físicos en los últimos 5 años.

c) El $83.33 \%$ de las personas que completaron el cuestionario no conocen desde qué año se realizan respaldos en sus dependencias. (Figura 3)

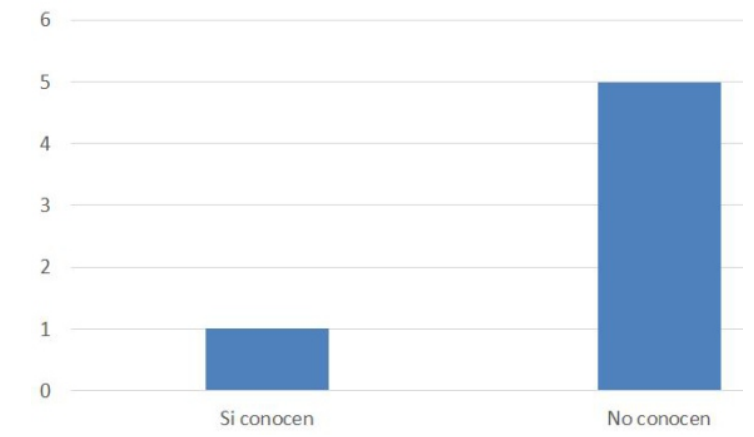

Figura 3. Conocimiento de la realización de respaldos de su dependencia.

d) $83.3 \%$ no conocen plataformas de software para recopilar, gestionar, difundir y preservar los documentos digitales. (Figura 4)

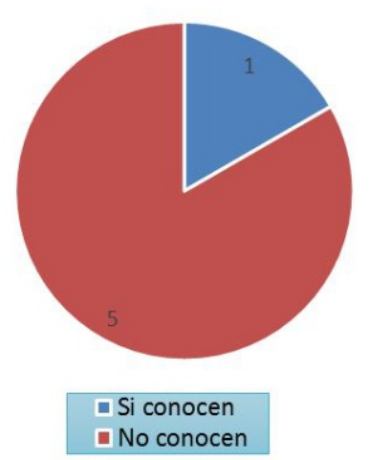

Figura 4. Conocimiento de sottware para almacenamiento de documentos digitales. e) El 100\% de las personas encuestadas están de acuerdo en que se debe implementar una plataforma o sistema de almacenamiento y preservación de documentos. (Figura 5)

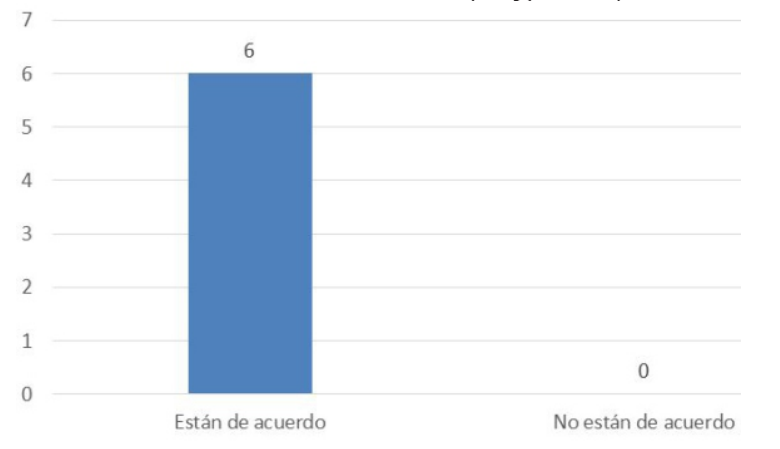

Figura 5. Implementación de software para almacenamiento de documentos digitales.

\section{DISCUSIÓN}

En el punto a se menciona que el $66.6 \%$ de las personas que completaron el cuestionario realizan respaldos de los documentos digitales a través de USB, CD, DVD y/o en la misma computadora, el $33.4 \%$ no realizan respaldo alguno, evidenciando que, de cada seis personas dos personas no realizan ningún tipo de respaldo digital a documentos de sus dependencias $u$ oficinas.

En el punto b se indica que el $16.67 \%$ de las personas que completaron el cuestionario archivan los documentos físicos producidos en los últimos 5 años, observando un déficit en el archivamiento de documentos físicos que pone en evidencia una problemática enquistada en oficinas y dependencias de la Vicepresidencia de Investigación de la UNJ en cuestión de administración y gestión de archivos.

En el punto c se menciona que el $83.33 \%$ de las personas que completaron el cuestionario no conocen desde qué año se realizan respaldos en sus dependencias, mostrando que de cada seis personas cinco desconocen los respaldos realizados de los documentos existentes en sus oficinas o dependencias, es decir que si se requiere documentos que data de años atrás sería difícil determinar su ubicación. 
En el punto $\mathbf{d}$ se indica que el $83.3 \%$ no conocen plataformas de software para recopilar, gestionar, difundir y preservar los documentos digitales, mostrando un inconveniente que se solucionará planificando capacitaciones a las personas que estarán relacionadas directamente con el repositorio digital.

En el punto e se menciona que el $100 \%$ de las personas encuestadas están de acuerdo en que se debe implementar una plataforma o sistema de almacenamiento y preservación de documentos; demostrando la importancia de este trabajo de investigación con la instalación e implementación de Dspace como repositorio digital.

Es importante mencionar que en las prerrogativas observadas de este trabajo de investigación, se coincide con lo escrito por Duperet Cabrera, Pérez Martínez, Cedeño Rodríguez, Ramírez Mustelier, \& Montoya Acosta (2015) en la necesidad de contar con un repositorio digital para la difusión, gestión y preservación de trabajos de investigación, igualmente cabe indicar que en esta investigación se evidencia de forma tangible la debilidad existente en la preservación de documentos de investigación y cualquier otro tipo de documentos como también el desinterés de la instalación, implementación y formalización de un repositorio digital en las Instituciones, haciendo hincapié en el fomento de instalación e implementación de repositorios digitales para formar estándares y herramientas que permitan la fácil difusión de trabajos de investigación en todo el mundo a través de la web, coincidiendo con lo mencionado por parte de Texier, De Giusti, Oviedo, Villarreal, \& Lira (2012).

\section{CONCLUSIONES}

Por lo antes expuesto se llega a la conclusión la viabilidad de utilizar Dspace como repositorio digital, por ser software libre y no exigir ningún pago para ser instalado, contener un entorno y
Igualmente, este artículo busca concientizar y ser un aporte para las instituciones que tienen la necesidad de preservar, gestionar y difundir su documentación en forma digital a través de la web, sobre todo en el ámbito científico.

Es preciso mencionar que este trabajo pretende ser tomado en cuenta como alternativa por las Universidades en proceso de licenciamiento, puesto que una de las exigencias de SUNEDU para cumplir con los indicadores establecidos, es contar con un repositorio digital para alojar trabajos de investigación como tesis de pregrado y post grado.

Es necesario investigar experiencias similares en otras instituciones con documentos administrativos, académicos y de investigación, porque podrían surgir otras propuestas viables con las expectativas institucionales que permitan la preservación y almacenamiento de documentos digitales.

\section{AGRADECIMIENTO}

A la Universidad Nacional de Jaén, en especial a la Oficina de Vicepresidencia de Investigación presidida por el Ph.D Manuel Antonio Canto Sáenz, por el compromiso con la investigación y las facilidades brindadas para llevar a cabo la implementación de Dspace en esta casa de estudios.

\section{BIBLIOGRAFÍA}

ALICIA - CONCYTEC. (08 de Junio de 2016). CONCYTEC. Recuperado el 05 de Diciembre de 2016, de ALICIA $\begin{array}{lllllllll}C & \mathrm{O} & \mathrm{N} & \mathrm{C} & \mathrm{Y} & \mathrm{T} & \mathrm{E} & \mathrm{C} & \text { : }\end{array}$ http://portal.concytec.gob.pe/images/do cumentos/alicia/directrices_repositorio. pdf

ALICIA. (21 de setiembre de 2016). ALICIA. Recuperado el 29 de diciembre de 2016, de ALICIA: https://sites.google.com/a/concytec.gob.pe/alici a/implementacion-de-repositorios/instalacion-yconfiguracion-de-repositorios/dspace 
Chazarra, J., Requena, V., \& Valverde, S. (28 de octubre de 2010). Facultad de Informática Universidad Complutense de Madrid. Recuperado el 30 de noviembre de 2016, de Universidad Complutense de Madrid: http://eprints.ucm.es/11078/1/MemoriaSI.p df

Duperet, E., Pérez, D., Cedeño, M., Ramírez, A., \& Montoya, L. (2015). Importancia de los repositorios para preservar y recuperar la información. Revista Médica de Santiago de Cuba, 19(10).

DURASPACE. (23 de diciembre de 2016). DURASPACE. Recuperado el 2016 de diciembre de 28, de DuraSpace wiki: https://wiki.duraspace.org/display/DSDOC $5 \mathrm{x} /$ Installing + DSpace

Ibai Sistemas S.A. (2012). Informe de adaptación de DSpace a Europeana. Madrid.

Polanco, J. (15 de Abril de 2016). Universidad de Costa Rica. Obten ido d e ht t ps:// ucrindex.ucr.ac.cr/: https://ucrindex.ucr.ac.cr/docs/repositoriosdigitales-definicion-y-pautas-para-sucreacion.pdf

Rodríguez Gairín, J. M., \& Sulé Duesa, A. (Junio de 2008). Facultat de Biblioteconomia $i$ Documentació Universitat de Barcelona. Recuperado el 10 de 11 de 2016, de Textos universitaris de biblioteconomia $i$

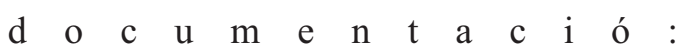
http://bid.ub.edu/20rodri2.htm

Silva, A. (27 de Enero de 2015). SEO Basico. Obtenido de http://www.seobasico.com/: http://www.seobasico.com/cuantasbusquedas-hacen-google/

Texier, J., De Giusti, M., Oviedo, N., Villarreal, G. L., \& Lira, A. (18 de Octubre de 2012). Repositorio Institucional de la UNLP. Recuperado el 25 de Enero de 2017, de h t t p : / / s edici.un l p. ed u a r / : http://sedici.unlp.edu.ar/bitstream/handle/1 0915/22943/Documento_completo.pdf?seq uence $=4$

\section{CORRESPONDENCIA}

Mack Johan Tarrillo Herrera

Los Laureles No 999 - Jaén - Cajamarca Perú.

mack_tarrillo_1@hotmail.com 